\title{
IDENTIFICACIÓN DE ESTRATEGIAS DE APRENDIZAJE EN EDUCACIÓN INFANTIL Y PRIMARIA: PROPUESTA DE INSTRUMENTOS
}

\section{IDENTIFICATION OF LEARNING STRATEGIES IN EARLY CHILDHOOD AND PRIMARY EDUCATION: A PROPOSAL OF INSTRUMENTS}

\author{
Honorio Salmerón*, Luis Ortiz Jiménez** y Sonia Rodríguez Fernández* \\ Universidad de Granada
}

\begin{abstract}
RESUMEN
El artículo contiene fundamentos y propuestas para la elaboración de pruebas sencillas que los profesores de aula pueden utilizar o adaptar para identificar en sus alumnos/as estrategias que utilizan cuando han de resolver tareas. También se presentan pruebas capaces de identificar en los profesores sus comportamientos estratégicos cuando actúan como docentes y como aprendices.
\end{abstract}

Palabras clave: Estrategias de aprendizaje, Educación Infantil y Primaria, instrumentos de evaluación.

\section{ABSTRACT}

This paper contains theoretical basis and practical proposals for classroom teachers to elaborate easy tests, when trying to identify students `strategies to solve academic tasks. We do also present tests which allow teachers to identify their own behaviors as both teachers and learners.

Key words: learning strategies, infant education, basic educacion, evalualtion's instruments.

\section{Introducción}

A partir de la revisión de los estudios sobre estrategias de aprendizaje, nos adherimos, para la etapa en la que estamos trabajando, a un concepto fundamentalmente relacionado con la toma de decisiones durante el proceso de realización de tareas de tipo intelectual (Nisbet, 1987), o una toma de decisiones consciente e intencional Monereo (1997).

* Profesores del Dpto. Métodos de Investigación y Diagnóstico en Educación de la Universidad de Granada.

** Licenciado en Psicopedagogía y doctorando de la Universidad de Granada. 
Focalizamos el objeto de nuestro trabajo en colaborar con las organizaciones en la educación de ciudadanos que deberían aprender razonando y ser, cada vez más, autónomos para aprender por sí mismos.

Ese proceso de toma de decisiones se fundamenta en primer lugar en la interacción de dos conocimientos: el que posee cada sujeto acerca de las habilidades académicas y cognitivas que se requieren para realizar una tarea y el autorreconocimiento de las que se poseen para ello, esto es, la autoconsciencia de los propios procesos de aprendizaje, o metacognición si seguimos a Flavell (1970).

En segundo lugar, pero en el mismo espacio-tiempo, el resultado de dicha interacción va a ser influido por: la percepción de las condiciones ambientales-contextuales propiciadas desde las actuaciones docentes, las características de la tarea en concreto, el valor que en cada momento el aprendiz otorga a la demanda y el resultado de la evaluación cognitiva que éste realiza de las consecuencias de experiencias anteriores semejantes.

Por tanto, un comportamiento estratégico se distinguirá por la capacidad de análisis de sí mismo y de situaciones, tareas, problemas, para responder conscientemente ante las demandas planteadas en un contexto determinado.

Todas estas acciones se dirigen en su conjunto hacia una finalidad y son, por tanto, más que una suma de secuencias o unión de habilidades.

Respecto a la tipología y clasificación de estrategias, no existe un acuerdo generalizado por la relativa juventud del campo de estudio (Weinstein y Meyer 1998); una de las primeras clasificaciones la hacen Resnick y Berc (1976) estableciendo estrategias generales y estrategias mediacionales.

Baron (1978) establece tres grupos principales de estrategias. En un primer grupo incluye las estrategias encargadas de buscar en la propia memoria respuestas relacionadas con un nuevo problema (formulación de cuestiones). Un segundo grupo estaría compuesto por aquellas encargadas de analizar los estímulos, dividiendo la tarea en pasos o apartados (cercana a la planificación) La tercera categoría se referiría a las estrategias que denomina de verificación.

Sternberg (1983) distingue entre estrategias ejecutivas (las usadas para planificar, controlar y revisar) y no ejecutivas (las no explícitas al realizar la tarea pero que están implicadas).

Kirby (1984) clasifica las estrategias de aprendizaje atendiendo a la especificidad de las habilidades implicadas; habla así de macroestrategias (más generalizables) y microestrategias (más específicas de cada tarea).

Weinstein y Mayer (1986) establecen una clasificación, a la que nos adherimos, que comprende estrategias de ensayo o repetición, estrategias de elaboración, estrategias de organización, estrategias de regulación o metacognitivas y por último un grupo de estrategias ligadas a los aspectos afectivo-motivacionales.

Estos autores intentaron formalizar un catálogo preliminar en torno a una serie de categorías de actuación. Cada una de éstas constaba de procesos y técnicas que, utilizadas por los estudiantes, pudieran descubrir la forma de adquirir la información, las formas de integrarla, y en su posterior recuperación. 
En este sentido, un paso previo a cualquier intento de evaluar las estrategias que usa una persona al enfrentarse a la realización de una tarea de aprendizaje es, definir qué fases o componentes debe tener esa actuación para que pueda ser definida como tal.

Siguiendo a autores como Brown (1987), podemos destacar tres grandes fases de una actuación estratégica: Planificación, regulación y evaluación.

Las actividades de planificación, previas a la ejecución de la tarea, incluyen aspectos como: identificación de la demanda, clarificación de objetivos, metas del aprendizaje, enfoques de la tarea, reconocimiento de conocimientos previos sobre la tarea, anticipación de tiempo de ejecución, selección de una forma (método) que nos pueda llevar a la solución, características del contexto (principalmente el generado por el demandante de la tarea), disponibilidad y selección de recursos básicos...

La regulación, en la realización de la tarea, incluye aspectos como la autodirección, organización del material adecuadamente, búsqueda de medios alternativos, seguimiento del plan trazado, ajuste de tiempos y esfuerzos, cumplimentación de las distintas partes de la tarea...

Finalmente, la evaluación, está referida a la autorevisión del proceso seguido; es la valoración sobre la consecución de los objetivos de la tarea; valoración de la calidad del resultado; decisión sobre cuando se ha terminado la tarea; corrección de los posibles errores cometidos; satisfacción por la superación de dificultades; valoración cognitiva de las consecuencias de todo el proceso; aplicaciones para futuras tareas.

\section{Modelos de instrumentos}

Para la identificación de las Estrategias de Aprendizaje, han sido utilizados un amplio grupo de instrumentos, pudiendo encuadrarlos en cuatro grandes bloques:

- Autoinformes, tanto generales como de tareas específicas.

- Observación.

- Entrevista, principalmente semi-estructurada.

- Cuestionarios.

Junto a estos métodos también se ha utilizado el análisis de ejecuciones (Nisbet, J., 1987).

Con respecto a cuestionarios, C. Vizcarro y otros (2000) realizan un resumen de los mismos, de los cuales significamos los siguientes:

- ACH. Caballero, A. (1972).

- SPQ. J.B Bigs (1978).

- LASSI. Weinstein, C.E. y otros (1983).

- IDEE. Selmes (1988).

- MSLQ. Pintrich y ortos (1991).

- IDEA. Vizcarro, C. y otros (1996).

- ACRA, Roman y Gallego (1994). 
Asimismo referidos a la actuación docente, aunque no traten de forma explícita la identificación de las estrategias que usan los docentes, podemos citar:

- Pautas de actuación docente. Alonso Tapia (1991).

- PIELE-PA. Hernández y Santana (1987).

\section{Nuestros planteamientos}

Acogiéndonos a estos fundamentos, construimos nuestros instrumentos para identificar las estrategias que usan tanto el alumnado en su proceso de aprendizaje como el profesorado al diseñar las acciones educativas que propicien el desarrollo de las mismas.

A partir de los antecedentes expuestos, nos decantamos por un proceso de identificación de estrategias de enseñanza y aprendizaje que se sitúen bajo un modelo ecológico situacional.

Según los estudios analizados observamos como una actuación estratégica tiene que tener en cuenta todos los elementos que intervienen en el proceso educativo, especialmente el conjunto relacional que se establece en el ecosistema aula. El profesorado transmite una serie de mensajes, que son percibidos por el alumnado de distintas formas, produciéndose en este intercambio una serie de adaptaciones mutuas.

Por otra parte las características de las tareas y las peculiaridades personales condicionarán igualmente las actuaciones a seguir por parte de unos y otros.

No podemos, consecuentemente, establecer un único método evaluativo, si pretendemos una inserción curricular comprensiva de las estrategias de aprendizaje. Para ello es necesario generar instrumentos que identifiquen el uso de las mismas desde el propio programa educativo en todo su desarrollo, desde la planificación a la evaluación.

Nuestra pretensión es acercar esos métodos evaluativos a la práctica educativa cotidiana de manera que puedan ser convenientemente adaptados al contexto que se pretende estudiar y mejorar.

Necesitamos pues instrumentos que reúnan las siguientes características:

$1^{\circ}$. Que puedan ser aplicados fácilmente sin producir una excesiva intrusión en la vida del aula y, por tanto, puedan ser usados por los propios responsables, como si se tratara de una actividad más, sin que se precise la presencia de un especialista.

$2^{\circ}$. Que no sean costosos tanto en referencia al tiempo de aplicación como en la confección o adquisición de material.

$3^{\circ}$. Por último, que sean fácilmente interpretables por el evaluador, aún cuando no ha sido aplicado directamente por él.

En suma, y para identificar las estrategias que usa el alumnado, nos inclinamos por la confección de tareas que integren para su correcta ejecución el seguimiento de unas fases lógicas: Planificación, regulación de la ejecución y evaluación (Brown, 1987).

Al mismo tiempo, y para el profesorado, procuraremos que los ítems que se refieren a actuaciones consideradas como de enseñanza estratégica no sean fácilmente identificables por el profesorado, para evitar el sesgo que provocaría la deseabilidad social. 


\section{Propuesta de instrumentos}

\section{A. Destinados al profesorado}

Con respecto al profesorado, nos inclinamos por realizar dos tipos de pruebas: Una consistiría en un cuestionario desde el que se le plantean una serie de acciones que deberían habitualmente en el aula, para que indique con que frecuencia las lleva a cabo.

La segunda prueba será un autoinforme con formato de entrevista semiestructurada. El entrevistador se limitará a dinamizar la realización de esa autoreflexión, proporcionando una serie de pistas que guíen el discurso del entrevistado.

A continuación detallamos estas dos propuestas:

\section{A.1. Cuestionario para la identificación de estrategias de enseñanza en el profesorado}

\section{Marco teórico}

La finalidad de este cuestionario es identificar las estrategias de enseñanza utilizadas por el profesorado, para promocionar el uso de estrategias de aprendizaje en sus alumnos/as.

Las pautas de actuación docente, generan en el alumnado una serie de prácticas que inciden de forma esencial en la forma en que se afronta el aprendizaje, presente y futuro. El clima en que se desarrollan las clases, los agrupamientos u organización social del trabajo y los modelos de evaluación utilizados, son aspectos que marcan al alumnado, y consecuentemente, producen unos efectos, positivos o negativos casi nunca neutros, en el desarrollo motivacional intrínseco al aprendizaje. De tal manera que, consciente o inconscientemente, hacen que ante situaciones análogas, desarrollen rutinas de comportamientos similares.

Por parte de distintos autores (Alonso Tapia, 1993, 1997; Hernández y Santana, 1988; Beltrán, 1991, 1993, 1998) se han desarrollado trabajos tendentes a identificar determinados aspectos de la actuación docente, su motivación, estilos, etc. Con el cuestionario que presentamos, se pretende identificar actuaciones estratégicas, entendidas éstas como un proceso de toma de decisiones acerca de los pasos a seguir para resolver una tarea, teniendo en cuenta para ello, las propias características de la misma, las características individuales y el entorno en que se realiza. Ello conlleva que el alumnado adquiera para sí estas formas de actuar, esto es, sean estratégicos en su aprendizaje o dicho de otra forma, aprendan como consecuencia de pensar. (Nisbet, 1987; Brown, 1987; Monereo, 1993, 1997; Pérez Cabaní, 1997; Weinstein y Meyer, 1998; Pozo y Monereo, 2000).

El resultado de la ejecución de la tarea adquiere mayor sentido cuando es construido por el alumno/a, tanto si es correcto como erróneo; en este último caso la mediación debe propiciar los apoyos necesarios para que el alumno /a sea capaz de identificar dónde, cómo y porqué del mismo y reflexione sobre el proceso seguido de manera que esa actividad autoreflexiva o de autocontrol generada, sea a su vez fuente de aprendizaje. 


\section{A.1.1. Estructura del Cuestionario y DEFinición DE VARiables}

Correspondiendo al modelo de actuación estratégica adoptado hemos confeccionado una estructura en torno a tres dimensiones: Planificación, Regulación (ejecución) y Evaluación (ver Cuadro 1).

\section{CUADRO 1: Distribución de los ítems en las dimensiones donde se incluyen.}

\begin{tabular}{|l|c|c|}
\hline \multicolumn{1}{|c|}{ Dimensión/estrategia } & Items & $\mathbf{N}^{\mathbf{0}}$ total de items \\
\hline Planificación & $1,2,3,4,5,6,7,8,9,10,11,12$ & 12 \\
\hline Regulación & $\begin{array}{c}18,19,20,21,22,23,24,25,26,27, \\
28,29,30,31,32,33,34,35,36\end{array}$ & 19 \\
\hline Evaluación & $\begin{array}{c}37,38,39,40,41,42,43,44,45,46, \\
47,48,49,50\end{array}$ & 14 \\
\hline Agrupamientos en clase & $13,14,15,16,17$ & 5 \\
\hline
\end{tabular}

Planificación: Consta de 12 ítems codificados como P1 a P12 (1 a 12).

Los ítems se han construido relacionándolos con una serie de actividades preparatorias, esto es, ponerse en disposición de ...

Las actividades de planificación, previas a la actuación, incluyen aspectos como: Clarificación de objetivos, metas de aprendizaje, enfoques en la realización de la tarea, reconocimiento de conocimientos previos sobre la tarea, tiempos, selección de forma (método) que nos pueda levar a la solución, identificación de la demanda, características del contexto (especialmente el generado por el demandante). Es el momento en el que el profesorado ha de determinar las necesidades del alumnado, explicitar la utilidad o funcionalidad del aprendizaje que se va a acometer, diseñar las tareas, decidir los métodos más idóneos de afrontarlas, anticipar los cambios formativos que provocarán, y establecer criterios claros para la valoración de las ejecuciones.

Regulación: consta de 19 ítems codificados como R1 a R19 (18 a 36). Es la fase de realización de la tarea, e incluye aspectos como la autodirección, organización adecuada del material, búsqueda de medios alternativos de solución, seguimiento del plan trazado, ajuste de tiempos y esfuerzos.

Una vez planificada la ejecución de a tarea, se pone en práctica considerando los diferentes ritmos que se siguen, observando como se completan las pautas establecidas, identificando de las operaciones realizadas, las ayudas que se precisan y los agrupamientos mas adecuados a la finalidad de la tarea.

Evaluación: consta de 14 ítems codificados como V1 a V14 (37 a 50). Hace referencia a valoración del proceso seguido, de la consecución de objetivos de la tarea, corrección de los posibles errores, satisfacción por la superación de dificultades, aplicabilidad del proceso en otras tareas...

Si bien no se debe afirmar que una fase sea más importante que otra en una secuencia estratégica, no es menos cierto que durante la fase de evaluación, o control del proceso 
como también se le puede denominar, es en la que nos jugamos gran parte del éxito del trabajo realizado. El docente ha de construir una cultura autoevaluativa en el aula de tal forma que los alumnos sean capaces de apreciar comprensivamente lo que hacen; si ésta valoración responde a los criterios y planteamientos establecidos en la planificación, no solo el aprendizaje declarativo sobre lo tratado será importante, sino que el factor motivacional se verá fortalecido, así como el desarrollo de hábitos de trabajo basados en formas estratégicas de pensamiento.

Por tanto el docente durante esta fase cuidará los mensajes que da a sus alumnos/as, reflexionando con ellos sobre los progresos alcanzados, la autovaloración de los trabajos, justificación y bondad de las decisiones adoptadas y las posibles transferencias del aprendizaje conseguido.

Organización social del aula: 5 ítems codificados como Q1 a Q5 (13 a 17). Se pretende identificar con estas acciones el formato preferido por el profesorado para organizar socialmente el trabajo del aula.

\section{A.1.2. NORMAS PARA LA ADMINISTRACIÓN}

Formato de las preguntas

El profesorado debe ser capaz de seleccionar y proponer las tareas más coherentes para desarrollar las estrategias que pretende (Nisbet, 1987; Monereo y Castelló, 1997). En base a esa capacidad, si le proponemos preguntas de respuesta dicotómica, su decisión al responder estará inducida por lo que se debería hacer más que por lo que realmente hace.

Por ello consideramos más conveniente formular preguntas que informen, sobre todo, de la frecuencia con que realizan una serie de comportamientos directamente relacionados con la actividad docente.

A través de estos datos se pueden identificar si realmente son estratégicos en sus actos, y por ende, como afirman (Nisbet, 1987; Monereo, 1993, 1997, Pérez Cabaní 1997; Weinstein y Meyer, 1998, Pozo y Monereo, 2000), provocarán en sus alumnos/as, por modelamiento, un comportamiento estratégico.

Formato de respuestas

Las respuestas se pueden recoger en escalas tipo LIKERT con cuatro posicionamientos de valores: 1 nunca, 2 pocas veces, 3 frecuentemente, 4 muy frecuentemente.

Condiciones de aplicación

Puede ser colectiva o individual pero en una sesión, si bien puede ser aconsejable entregar el cuestionario y recogerlo transcurrido un tiempo que permita la reflexión sobre las cuestiones planteadas en los ítems.

No se establece un tiempo determinado para contestar el cuestionario. 


\section{A.1.3. CARACTERÍSTICAS TÉCNICAS}

Validez del cuestionario

El cuestionario fue sometido a un proceso de validación de contenido para pruebas criteriales de aula (Hambleton, R.K. 1984) a partir del índice de congruencia entre jueces que fueron: tres en Psicología de la Educación, tres en Pedagogía y tres maestros con amplia experiencia.

A todos se le suministraron tres copias del cuestionario para que valorasen cada ítem en relación a los tres criterios siguientes:

$1^{\circ}$. Coherencia (C): Entendida como la cantidad de relación que encuentran entre el ítem y la dimensión estructural en la que se incluye.

$2^{\circ}$. Representatividad (R): donde informan de la bondad del ítem entre todos los posibles considerando el ámbito del currículum desarrollado.

$3^{\circ}$. Calidad Técnica (CT): referida a la calidad del lenguaje utilizado en la redacción del ítem para no inducir a error, por sesgos gramaticales, al que contesta.

Adoptamos el acuerdo de eliminar, en primer lugar, aquellos ítems cuya puntuación media obtenida en coherencia, fuera inferior a 6,50 en una escala de 1 a 9. En base a ello fueron eliminados cuatro ítems. Por similares motivos, de los ítems restantes, hubieron de reformularse tres que aunque obtuvieron puntuaciones suficientes en coherencia no fue así en representatividad; Los cincuenta ítems definitivos obtuvieron la suficiente calidad técnica (ver anexo 1).

Asimismo se les pidió que otorgaran una puntuación global al cuestionario (Cuadro 2) en función de tres aspectos del mismo con objeto de mejorar el formato del cuestionario:

- Extensión

- Presentación

- Facilidad para contestar

\section{CUADRO 2: Valoración global otorgada por los expertos al cuestionario.}

\begin{tabular}{|l|c|c|}
\cline { 2 - 3 } \multicolumn{1}{c|}{} & Media & Desv. Típica \\
\hline Extensión & 8,44 &, 537 \\
\hline Presentación & 8,33 &, 705 \\
\hline Facilidad para contestar & 8,33 &, 615 \\
\hline
\end{tabular}

\section{Fiabilidad}

Se ha llevado a cabo un análisis de fiabilidad referido a consistencia interna utilizando el Coeficiente Alfa de Cronbach, obteniendo un valor de 0,83. La muestra experimental de sujetos, en este ensayo piloto, estuvo formada por 75 maestros recién titulados en diferentes especialidades y 20 maestros en ejercicio en los niveles de Educación Infantil y Primaria de diferentes planes de estudio y años de experiencia.

\section{A.1.4. NORMAS DE PUNTUACIÓN E INTERPRETACIÓN}

Los datos obtenidos a través del cuestionario, se puntuarán de la siguiente forma:

Dicotomizamos los valores 1, 2, 3 y 4 de la escala likert asignando, a las repuestas 1 y 2 valor 0 , y a las 3 y 4 , valor 1 . Obtenemos las medias de estos valores para cada dimensión que equivaldrían al porcentaje de uso de las estrategias. 
Una limitación de la bondad de las respuestas se encuentra en el hecho de que el profesorado reflexiona sobre el cuestionario mientras lo contesta interaccionando la respuesta a cada cuestión con cada una de las siguientes respuestas. Sin embargo algunas ventajas se obtienen: Weinstein y Meyer (1998) comprobaron como, tras la aplicación de determinadas pruebas acerca de la metacognición y estrategias, mejoraba la planificación y el autocontrol de aquellos sujetos que se sometieron a las mismas.

\section{A.1.5. ANÁLISIS DE DATOS}

Para el adecuado tratamiento de los datos obtenidos por el cuestionario, puede usarse el paquete informático SPSS Versión 9.0.

\section{A.2. Autoinformes del profesorado}

Se han utilizado en formato de entrevistas semi-estructuradas. Se realizaron a maestros en ejercicio de forma individual respondiendo a un guión general en torno al cual se mantiene una conversación con el entrevistado.

Pretendimos identificar dos aspectos diferentes con esta entrevista: por una parte sus actuaciones individuales cuando ejerce como aprendiz, por otra su propia percepción como docente.

El guión general fue el siguiente:

E.1. Motivación, como aprendiz-docente.

E.2. Autoeficacia cuando ejerce como aprendiz.

E. 3. Autopercepción cuando aprende o consciencia de los procesos que usa.

E.4. Necesidad de cambios en su propio proceso de aprendizaje

E.5. Nivel de conocimiento sobre las potencialidades de sus alumnos.

E.6. Sensibilidad a los cambios (positivos y negativos) observados en las actitudes del alumnado.

E.7. Grado de satisfacción de su actuación docente.

E.8. Posibles mejoras en sus actuaciones docentes.

E.9. Nivel de conocimiento sobre formas de desarrollar en el aula programas de enseñar a aprender pensando.

E.10. Necesidades para desarrollar un programa de enseñar a pensar en su aula

Las anotaciones se hicieron a posteriori, inmediatamente tras finalizar la entrevista.

En un principio, entendemos que no es preciso utilizar ningún paquete informatizado para interpretar los datos obtenidos, ya que la entrevista no es excesivamente larga. No obstante, según los casos, y si se trata de analizar una población amplia, toda la plantilla de un centro, se pueden usar el paquete informático AQUAD o similar.

Nos interesarán aquellos datos que respondan a las siguientes características:

- Ideas que más se repiten

- Ideas originales y llamativas

- Opiniones más controvertidas 


\section{B. Instrumentos para el alumnado}

\section{B.1. Identificación de estrategias de aprendizaje en niños pequeños: el laberinto}

Utilizamos una prueba de papel y lápiz con formato de juego que podría ser cumplimentado en mayor o menor medida por todos y de la que podíamos obtener información acerca de las tres dimensiones citadas.

Esta tarea puede ser realizada tanto por el alumnado de educación infantil como por el del primer ciclo de primaria.

Esta sería su FICHA TÉCNICA:

Aplicación: Niños entre 3 y 8 años

Organización: Grupos de 2 alumnos.

Tiempo de administración: 20 minutos (máximo).

Técnicas de análisis: Observación, verificación de tareas.

Recursos: • Grabadora, vídeo.

- Hoja de registro (ANEXO II).

\section{Descripción de la tarea:}

Se trata de que resuelvan un laberinto, usando, para trazar el camino, distintos elementos: punzón, bolitas de papel, lápiz de color, etc., a elegir por el sujeto.

\section{Normas de administración:}

Las limitaciones de este alumnado para leer, comprender y responder condiciona el tipo de prueba. También la presencia de una persona ajena al aula genera extrañeza y puede propiciar que los niños no la cumplimenten en las mejores condiciones. Por ello recomendamos que sea el propio responsable del aula quien realice la administración.

Teniendo en cuenta estos aspectos, consideramos que el mejor administrador de la prueba era el profesorado de aula lo que exigió que realizáramos, con ellos, sesiones preparatorias de entrenamiento para dotarles de unas pautas generales acerca de cómo actuar y qué datos serían los más importantes a observar. A partir de estas consideraciones, la administración de la prueba debe seguir básicamente el siguiente proceso:

1. El profesor selecciona o diseña un laberinto de dificultad adaptada para el nivel cognitivo que corresponde a su alumnado.

2. Conviene explicitar claramente la tarea a realizar y realizar algunos ejemplos prácticos.

3. A continuación se les presenta un nuevo laberinto, paralelo al anterior.

4. Una vez resuelta la tarea, es conveniente que los niños se relajen, decorando su trabajo.

5. La prueba finaliza pidiéndoles a cada uno, que verbalicen opiniones sobre el trabajo que han realizado en relación a: Satisfacción por la tarea, facilidad - dificultad de ejecución, cambios motivacionales para situaciones de aprendizaje posteriores semejantes.

\section{Datos relevantes para la interpretación:}

- Atención durante las explicaciones. 
- Disposición al iniciar la tarea (como colocan el papel, el material a usar, etc.).

- Lugar de inicio en la resolución del trabajo (por la salida o por el centro del laberinto, etc.).

- Acciones previas que realiza (marcar con lápiz para luego punzar, colocar bolitas, etc.).

- Orden lógico en la ejecución del trabajo.

- Comentarios que realiza durante el trabajo.

- Correcciones o modificaciones que realizan durante la ejecución.

- Variables expresadas anteriormente en el momento de finalizar el trabajo.

\section{Normas de puntuación:}

Se anotará «si» o «no» en la casilla correspondiente de la hoja de registro (ver anexo 2) a cada uno de los comportamientos que se consideran incluidos en una actuación estratégica.

A las anotaciones «Si» se les otorgará el valor uno y a las anotaciones «No» valor cero.

Asimismo se valorará la calidad del trabajo con una puntuación entre: 1 (tiene varios inicios en el laberinto no concluidos ninguno con nivel de suciedad alta); 2 (iguales indicadores de suciedad pero con al menos un camino concluido); 3 (Un solo itinerario concluido pero con nivel de suciedad media-alta) y 4 (Un solo itinerario concluido y trabajo limpio).

\section{Normas de valoración y redacción de informe:}

Las puntuaciones medias que se obtengan se agruparán por fases (de planificación, de ejecución y de evaluación) obteniendo también una media global más otra puntuación por la calidad del trabajo. Estas cinco puntuaciones deben ser valoradas independientemente.

El informe, además de las puntuaciones obtenidas, debería incluir una gráfica representativa de las mismas.

Entenderemos que hay una forma de actuar estratégica a partir de aquellos casos cuyas puntuaciones superan el $75 \%$, en cada apartado y en el global. Asimismo, la puntuación de la calidad del trabajo considerada adecuada, deberá oscilar entre 3 y 4.

\section{B.2. Identificación de estrategias de aprendizaje en alumnado de los ciclos $2^{\circ} y 3^{\circ}$ de Educación Primaria: el juego de la isla}

Este alumnado ya ha debido desarrollar sus habilidades lectoescritoras y evolucionado en sus formas de actuar (han entrado en la fase de las operaciones concretas). Por lo tanto podemos aprovechar esta coyuntura y desarrollar pruebas a través de las cuales pueda plasmar sus propias ideas.

No podemos olvidar que el aspecto lúdico sigue siendo muy importante en esta etapa de desarrollo. Por ello deberíamos diseñar pruebas que comprendiesen estos aspectos, de manera que les resulte atractiva su ejecución y por tanto se impliquen más al cumplimentarla A la vez debe ser una prueba que no sea muy complicada, fácil de comprender, y que permita diferentes formas de expresión (lenguaje escrito y gráfico). Más tarde habrán de verbalizarlo. 
Con estos planteamientos iniciales nos inclinamos por seleccionar una prueba de supervivencia diseñada por F. Tonucci, a la que hemos dotado de una nueva significación. Se trata del Juego de la Isla. (ver anexo 3).

Se puede realizar de forma colectiva o individual en un tiempo máximo de 30 minutos.

Puede ser aplicada tanto por el responsable del aula como por un investigador externo.

Los criterios de corrección giran igualmente en torno a las actuaciones de planificación, ejecución y evaluación al resolver la tarea.

La planificación se valorará en función de las actividades previas para irse a la isla: que se llevaría...

Para valorar la ejecución se tendrán en cuenta las tareas que realizaría una vez llegado a la isla; debe ajustarse a lo que se llevó siguiendo unos procesos secuenciales lógicos.

Para las actividades de evaluación se tendrán en cuenta los dibujos realizados en función de cómo está la isla al llegar y cómo cuando pasen cinco años. Se tendrán en cuenta aquellas anotaciones que hagan en función del valor que crean que ha tenido su estancia en la isla (qué está bien, que cambiarían, etc.).

Se valorarán con «1» aquellas aportaciones que se consideren útiles (por ejemplo: semillas, etc.). Con «0» aquellas que no significan nada (por ejemplo: llevarse una televisión sabiendo que no hay luz eléctrica, o bien aplicar cosas que no se llevó en principio).

Se establecerán puntuaciones para cada dimensión, una media global y una puntuación por la calidad del trabajo entre 1 y 4 siguiendo los mismos criterios que para la prueba del laberinto.

\section{Referencias bibliográficas}

Alonso Tapia, J. (1991). Motivación y aprendizaje en el aula. Cómo enseñar a pensar. Madrid: Santillana.

Anderson, J. R. (1985). Cognitive psycology and its implications. San Francisco: Freman.

Brown, A. L. y Ferrara, R. (1983). Learning remembering and understanding. En J. Favell y E. Markman (comps). Handbock of child psichology. vol. 3, pp. 77-166.

Bigg, J. B. (1978). Individual and group differencies in study processes. British Jouunal of Educational Psycohology, 48, 266-279.

Buendía Eisman, L. y Colás, M. P. (1992). Investigación Educativa. Sevilla: Alfar.

Buendía Eisman, L. y Olmedo, E. (2000). Estrategias de aprendizaje y procesos de evaluación en la Educación universitaria. BORDON, vol. 52, n², pp. 151-163.

Buendía Eisman, L. (1997). Métodos de Investigación en Psicopedagogía. Madrid: McGraw-Hill.

Dansereau, D. y otros (1983). Learning strategies training: Effects of sequencing. Journal of Experimental Education, vol. 51(3), pp. 102-108.

Graham Gibbs (2000). Institutional learning and teaching strategies in English Higher Education. Higher Education, 40, pp. 351-372.

Hambleton, R. K. (1984). «Validating the test scores». En Berk, R.A. (Ed.), A Guide to Criterion - referenced Test Construction, $2^{\mathrm{a}}$ Ed. Baltimore: The Johns Hopkins University Press.

Haywood, C. (1996). «Educación cognitiva temprana: una clave para el éxito escolar». En Molina García, S. (coord.) «Educación Cognitiva». Zaragoza: Mira editores. 
Hernandez, J. y Santana (1988). La educación de la personalidad: el papel del profesor. Barcelona: Oikos-Tau.

Mckeachie, W. J. y otros (1985). Teaching leraning strategies. Educational Psychologist, vol 20(3), pp. 153-160.

Monereo, C. y Castello, M. (1997). Las estrategias de aprendizaje: como incorporarlas a la práctica educativa. Barcelona: EDEBE

Monereo, C. (1993). Profesores y alumnos estratégicos: cuando aprender es consecuencia de pensar. Madrid: Pascal.

Muria Vila, I. y otros (1990). Entrenamiento en estrategias preinstruccionales y de aprendizaje cognoscitivas en la enseñanza de contenidos de psicofisiología. Revista Mexicana de Psicología, vol. 7(1-2), pp. 57-64.

Nisbet, J. y Shuksmit, J. (1987). Estrategias de aprendizaje. Madrid: Santillana.

Novak y Gowin (1988). Aprendiendo a pensar. Barcelona: Martínez Roca.

Pagano, R. (1998). «Estadística para las ciencias del comportamiento». México: Thomson.

Pérez Cabani, M. L. (coord.) (1996). La enseñanza y el aprendizaje de estrategias desde el currículum. Universidad de Girona: Horsori.

Pozo, J. I. y Monereo, C. (coord.) (2000). El aprendizaje estratégico. Madrid: Santillana.

Román, J. M. y Gallego, S. (1994). ACRA, Escalas de estrategias de aprendizaje. Madrid: TEA

Scannapieco, F. (1997). Formal debate: An Active Learning Strategy. Journal of Dental Education, vol. $61, \mathrm{n}^{\circ} 12$, pp. $55-61$.

Selmes, I. (1988). Inventario del estudio en la escuela IDEE. En Selmes, I.: La mejora sobre las habilidades para el estudio. Madrid: Paidos/MEC.

Sternberg, R. (2000). Como desarrollar la inteligencia práctica en la escuela. Madrid: Santillana.

Sternberg, R. J. y Spear-Swerling (2000). Enseñar a pensar. Madrid: Santillana.

Tait, H. y Entwistle (1996). Identifying students al risk through ineffective study strategies. Higher Education, 31, pp. 97-116.

Tonucci, F. (1989). Cómo ser niño. Barcelona: Barcanova.

Vermunt, J. (1996). Metacognitive, cognitive and affective aspects of learnign styles and strategies: A phenomenographic analysis. Higher Education, 31, pp. 25-50.

Vizcarro, C. y otros (2000). Evaluación de Estrategias de Aprendizaje. En Pozo y Monereo (2000): El aprendizaje estratégico. Madrid: Santillana.

Weinstein, C. E., Goetz, E. T. y Alexander, P. A. (1988) (comps.). Learning and study strategies: Issues in assessment, instruction and evaluation. Nueva York: Academic Press.

Weinstein, C. \& Meyer, D. (1998). «Implicaciones de la Psicología cognitiva en la aplicación de pruebas: contribuciones a partir del trabajo realizado en estrategias de aprendizaje». En Wittrock y Baker (comp.) (1998). Test y cognición. Madrid. Paidos.

Fecha de recepción: 23-VII-01

Fecha de revisión: 10-IX-01

Fecha de aceptación: 5-II-02 


\section{ANEXO 1 \\ Cuestionario para la identificación de estrategias de enseñanza en el profesorado}

A continuación te proponemos una serie de cuestiones relacionadas con los procesos de enseñanza en el aula.

Te pedimos que valores en qué grado utilizarías cada una de esas acciones, rodeando uno de los cuatro posicionamientos que se especifican para cada una, $(1,2,3$ y 4$)$, valores que se codifican respectivamente como se explica a continuación:

\begin{tabular}{|c|c|c|c|}
\hline 1 & 2 & 3 & 4 \\
\hline NUNCA & A VECES & CASI SIEMPRE & SIEMPRE \\
\hline
\end{tabular}

Puedes comenzar a partir de aquí.

\begin{tabular}{|c|c|c|c|}
\hline & & ITEM & \\
\hline 1 & P01 & $\begin{array}{l}\text { Anuncio con antelación el modo específico en que los alumnos/as deben afrontar } \\
\text { una tarea }\end{array}$ & $1-2-3-4$ \\
\hline 2 & P02 & Señalo las utilidades que puede tener la nueva materia a tratar & $1-2-3-4$ \\
\hline 3 & P03 & Realizo una introducción enlazando la nueva materia a tratar con la anterior & $1-2-3-4$ \\
\hline 4 & P04 & Proporciono un guión previo al iniciar un nuevo tema & $1-2-3-4$ \\
\hline 5 & P05 & Indico el procedimiento a seguir para una tarea determinada & $1-2-3-4$ \\
\hline 6 & P06 & Indico el modo en que hay que organizarse & $1-2-3-4$ \\
\hline 7 & P07 & Claramente indico las metas y objetivos que se persiguen con cada actividad & $1-2-3-4$ \\
\hline 8 & P08 & Sugiero las estrategias que ayuden al alumno a realizar una actividad & $1-2-3-4$ \\
\hline 9 & P09 & $\begin{array}{l}\text { Activo los conocimientos previos mediante preguntas para facilitar y conectar la } \\
\text { nueva información con la que ya poseen }\end{array}$ & $1-2-3-4$ \\
\hline 10 & P10 & Emito mensajes que ayuden a planificar la tarea & $1-2-3-4$ \\
\hline 11 & P11 & Señalo cuando y por qué usar una estrategia determinada & $1-2-3-4$ \\
\hline 12 & $\mathrm{P} 12$ & Digo: «Cuando terminéis esta tarea sabréis hacer ...» & $1-2-3-4$ \\
\hline 13 & Q01 & Utilizo el trabajo individual en la realización de las actividades & $1-2-3-4$ \\
\hline 14 & Q02 & Utilizo el grupo de dos alumnos/as para las actividades & $1-2-3-4$ \\
\hline 15 & Q03 & Utilizo el pequeño grupo ( 4 a 6 miembros) para realizar las actividades. & $1-2-3-4$ \\
\hline 16 & Q04 & Utilizo el grupo medio ( 7 a 12 miembros) para realizar las actividades & $1-2-3-4$ \\
\hline 17 & Q05 & Utilizo el gran grupo (toda la clase) para realizar las tareas. & $1-2-3-4$ \\
\hline 18 & R01 & $\begin{array}{l}\text { Inicio las clases presentando información nueva, sorprendente o incongruente con } \\
\text { los conocimientos previos para despertar el interés }\end{array}$ & $1-2-3-4$ \\
\hline
\end{tabular}




\begin{tabular}{|c|c|c|c|}
\hline & & ITEM & \\
\hline 19 & R02 & $\begin{array}{l}\text { Adecuo el ritmo de presentación de la nueva información para que los alumnos/as } \\
\text { la puedan ir asimilando }\end{array}$ & $1-2-3-4$ \\
\hline 20 & R03 & $\begin{array}{l}\text { Digo a los alumnos/as el modo específico en que deben estudiar para una tarea } \\
\text { evaluadora o un examen }\end{array}$ & $1-2-3-4$ \\
\hline 21 & R04 & $\begin{array}{l}\text { Ilustro las ideas con ejemplos o imágenes concretas que puedan servir al } \\
\text { alumno/a como referente para situarse ante la materia }\end{array}$ & $1-2-3-4$ \\
\hline 22 & R05 & En mis mensaje incluyo: «Esto caerá en el examen» & $1-2-3-4$ \\
\hline 23 & R06 & En mis mensajes incluyo: «Haced los ejercicios para ver quién es el mejor» & $1-2-3-4$ \\
\hline 24 & R07 & $\begin{array}{l}\text { En mis mensajes incluyo: «Si hacéis este ejercicio como os he enseñado sabréis } \\
\text { hacer ...» }\end{array}$ & $1-2-3-4$ \\
\hline 25 & R08 & Actúo como modelo de los procedimientos que aconsejo utilizar & $1-2-3-4$ \\
\hline 26 & R09 & $\begin{array}{l}\text { Durante la ejecución de una tarea: No doy indicaciones } \\
\text { NOTA: En esta pregunta las respuestas serán: } 1 \text { significa que no da indicaciones } \\
\text { y } 4 \text { que si las da muy frecuentemente }\end{array}$ & $1-2-3-4$ \\
\hline 27 & R10 & Durante la ejecución de una tarea: Indico lo que hace mal & $1-2-3-4$ \\
\hline 28 & R11 & Durante la ejecución de una tarea: Indico por qué está mal & $1-2-3-4$ \\
\hline 29 & R12 & $\begin{array}{l}\text { Durante la ejecución de una tarea: Indico como pueden corregirla si cometen un } \\
\text { error }\end{array}$ & $1-2-3-4$ \\
\hline 30 & R13 & $\begin{array}{l}\text { Ante las posibles dificultades: No contesto a sus preguntas } \\
\text { NOTA: En esta pregunta, } 1 \text { significa que no contesto y } 4 \text { que muy frecuentemente } \\
\text { contesto }\end{array}$ & $1-2-3-4$ \\
\hline 31 & R14 & Ante las posibles dificultades: Les digo que se esfuercen y piensen & $1-2-3-4$ \\
\hline 32 & R15 & Ante las posibles dificultades les doy la respuesta a los problemas & $1-2-3-4$ \\
\hline 33 & R16 & $\begin{array}{l}\text { Ante posibles dificultades, les ofrezco las pistas para que alcancen la respuesta } \\
\text { por sí solos }\end{array}$ & $1-2-3-4$ \\
\hline 34 & R17 & Les sugiero que tengan en cuenta los tiempos que van empleando & $1-2-3-4$ \\
\hline 35 & R18 & Propongo que dividan la tarea en pasos consecutivos & $1-2-3-4$ \\
\hline 36 & R19 & Los mensajes que emito durante la realización de la tarea son positivos & $1-2-3-4$ \\
\hline 37 & V01 & $\begin{array}{l}\text { Les pido a mis alumnos/as que reflexionen sobre como se sienten tras superar una } \\
\text { dificultad }\end{array}$ & $1-2-3-4$ \\
\hline 38 & V02 & $\begin{array}{l}\text { Tras realizar una tarea con mis alumnos/as, pasamos a la siguiente actividad sin } \\
\text { más mensajes } \\
\text { NOTA: En esta pregunta, las respuestas serán: } 1 \text { que no hay mensajes y } 4 \text { que } \\
\text { muy frecuentemente hay mensajes }\end{array}$ & $1-2-3-4$ \\
\hline 39 & V03 & $\begin{array}{l}\text { Cuando terminan una tarea, informo de modo global de la corrección o } \\
\text { incorrección de la tarea }\end{array}$ & $1-2-3-4$ \\
\hline 40 & V04 & Cuando termina una tarea, señalo los posibles aspectos incorrectos y mejorables & $1-2-3-4$ \\
\hline 41 & V05 & Cuando terminan una tarea, señalo las posibles vías de mejora & $1-2-3-4$ \\
\hline 42 & V06 & Hago hincapié en los progresos aunque el trabajo no esté totalmente bien & $1-2-3-4$ \\
\hline
\end{tabular}




\begin{tabular}{|c|c|l|c|}
\hline & & \multicolumn{1}{|c|}{ ITEM } & \\
\hline 43 & V07 & Incido en el proceso seguido para resolver una determinada actividad & $1-2-3-4$ \\
\hline 44 & V08 & Hago patente el aprendizaje que hemos conseguido después de realizar una tarea & $1-2-3-4$ \\
\hline 45 & V09 & Refuerzo las capacidades de los alumnos/as & $1-2-3-4$ \\
\hline 46 & V10 & $\begin{array}{l}\text { Considero que el sistema de evaluación que uso detecta el grado de adquisición } \\
\text { de la materia que ha sido enseñada }\end{array}$ & $1-2-3-4$ \\
\hline 47 & V11 & Utilizo distintos métodos para evaluar el mismo contenido & $1-2-3-4$ \\
\hline 48 & V12 & Tras la evaluación, explico la razón de pro qué la tarea no es correcta o mejorable & $1-2-3-4$ \\
\hline 49 & V13 & Pido que reflexionen sobre los procesos que han seguido para realizar la tarea & $1-2-3-4$ \\
\hline 50 & V14 & $\begin{array}{l}\text { Les manifiesto, a mis alumnos/as, que confío en sus capacidades para el } \\
\text { aprendizaje }\end{array}$ & $1-2-3-4$ \\
\hline
\end{tabular}




\section{ANEXO 2 \\ El laberinto}

\section{HOJA DE REGISTRO}

\section{ALUMNO/A}

EDAD

\begin{tabular}{|c|c|c|c|c|}
\hline & & SI & NO & Observaciones \\
\hline \multirow{7}{*}{ Planificación } & Tiene claros los objetivos de la tarea & & & \\
\hline & Reconoce las características de la tarea & & & \\
\hline & $\begin{array}{l}\text { Usa el medio más adecuado para resolver la tarea (eficaz y } \\
\text { útil). }\end{array}$ & & & \\
\hline & ¿Identifica las partes o fases? & & & \\
\hline & Sabe cuando habrá terminado la tarea & & & \\
\hline & Sabe cuando está bien y cuando no la tarea. & & & \\
\hline & Establece un plan de acción & & & \\
\hline \multirow{10}{*}{ Ejecución } & Usa el recurso (lápiz, bolitas, ceras, etc.) que eligió. & & & \\
\hline & Si cambia el recurso, ¿Da razones del cambio? & & & \\
\hline & Sigue el plan trazado & & & \\
\hline & Organiza el material adecuadamente & & & \\
\hline & $\begin{array}{l}\text { Realiza correcciones sobre la marcha para hacer mejor la } \\
\text { tarea (por ejemplo si cogió el punzó y comprueba que no le } \\
\text { es válido para la tarea) }\end{array}$ & & & \\
\hline & Es consciente de que va completando las fases de la tarea & & & \\
\hline & Pide que se le repitan las instrucciones. & & & \\
\hline & Pide que se le recuerden los colores que debe usar. & & & \\
\hline & Se vuelve a plantear interrogantes. & & & \\
\hline & Sabe cuando ha terminado & & & \\
\hline \multirow{10}{*}{ Evaluación } & Alcanza el objetivo planteado. & & & \\
\hline & Es capaz de afirmar si está bien o no. & & & \\
\hline & Es consciente de los posibles errores cometidos. & & & \\
\hline & Está satisfecho del resultado de la tarea & & & \\
\hline & $\begin{array}{l}\text { Puede relatar el proceso que ha seguido para completar la } \\
\text { tarea }\end{array}$ & & & \\
\hline & $\begin{array}{l}\text { ¿Haría el mismo proceso para hacer la misma tarea una } \\
\text { segunda vez? }\end{array}$ & & & \\
\hline & Puede identificar la parte más dificultosa de la tarea & & & \\
\hline & Cree que lo que ha hecho le puede servir para otros trabajos & & & \\
\hline & Le ha gustado la tarea que ha realizado & & & \\
\hline & Le ha parecido difícil. & & & \\
\hline
\end{tabular}




\section{ANEXO 3 \\ El juego de la isla}

Nombre:

Colegio: Curso:

Edad: Fecha:

Imagínate que debes trasladarte a una Isla desierta, donde no hay personas, ni animales, ni árboles; en donde se está bien y hace buena temperatura, donde el agua es buena y crece la hierba. Tú deberás irte con una embarcación para quedarte, imaginariamente, para toda la vida. Puedes llevarte lo que quieras.

¿QUÉ COSAS HARÁS CUANDO LLEGES A LA ISLA?

DIBUJA LA ISLA TAL Y COMO LA VES CUANDO LLEGUES.

DIBUJA LA ISLA COMO SERÁ DESPUÉS DE UN AÑO DE LLEGAR.

Cada apartado se contestará en una página independiente. 